\title{
Mechanical Links Between Locomotion and Breathing: Can You Breathe With Your Legs?
}

\author{
Hsueh-tze Lee and Robert B. Banzett \\ News in Physiological Sciences
}

$1997 ; 12 ; 273-278$

\begin{abstract}
Mechanical links between locomotion and respiration are inherently appealing because they may lower the cost of respiration, but they may also limit the ability to adapt ventilation appropriately to physiological needs. Although correlative evidence supports several linkage mechanisms, mechanical links contribute $<20 \%$ of the tidal volume in exercising animals.
\end{abstract}

Observations of consistent integer relationships between breathing and stride cycles in exercising birds and mammals date from Marey's observations in the last century. The tight temporal correlation between locomotor and respiratory cycles in animals such as hopping wallabies, galloping dogs and horses, and flying bats has led investigators to propose that during locomotion, muscles of exercising limbs provide some or all of the work of breathing. In this paper, we discuss the possible advantages and disadvantages of mechanical linkages between locomotion and breathing, consider mechanical mechanisms that could link these two activities, and describe experiments showing that the total effect of such linkages provide a fraction of tidal volume.

\section{Kinds of linkages}

Locomotion and breathing may be linked neurally or mechanically. Neural linkages exist: activity in limb motor nerves is coordinated with activity in respiratory motor nerves even during fictive locomotion in paralyzed decerebrated animals. In contrast, the extent of mechanical linkages is less certain. The observation that breathing and locomotion are coordinated does not necessarily imply a mechanical link. However, if mechanical interactions exist, proper neural coordination may be necessary for the animal to benefit from the interaction.

Mechanical interactions between breathing and locomotion may occur in either direction: respiratory muscle contraction could affect the locomotor cycle, or conversely, the locomotor cycle (stride or wingbeat) could contribute to respiratory flow, the focus of this review.

Although mechanical interactions have been proposed in a range of animals, including fish, lizards, birds and mammals, and during various forms of locomotion, from hopping in wallabies to rowing in humans, we limit our review to flying, hopping, walking, and running in birds and mammals.

\section{Advantages and disadvantages of mechanical links}

One possible advantage of a mechanical link between breathing and 
locomotion is reduction of the energetic cost of breathing. The best estimates of the oxygen cost of breathing during exercise have been made by asking human subjects to voluntarily mimic their respiratory patterns during exercise. These studies show that, during exercise at maximal aerobic capacity (VO2 max), the cost of breathing averages $10 \%$ of total oxygen consumption; during moderate exercise (70\% of maximal aerobic capacity), the cost of breathing is a smaller fraction of total oxygen consumption, $+/-5 \%$ (1). Transferring energy from limb muscles to breathing saves energy only if this energy would have otherwise been lost. In running (but probably not in flying), much energy is stored from cycle to cycle by alternating among gravitational potential, elastic, and kinetic forms. In breathing, a large part of the energy is expended to overcome airway and tissue resistance and dissipates as heat; little or none is available for the next cycle. Thus transfer of energy from locomotor activity to breathing might reduce the work of respiratory muscles but increase the work of the legs.

A second possible advantage is that breathing performance (maximum ventilation) may be increased with the assistance of limb muscle activity. Even in the absence of energy savings, this could be advantageous in cases when the power or endurance of respiratory muscles limits alveolar ventilation, causing a drop in alveolar PO2 during very heavy exercise. Such a limit to breathing performance has been observed in thoroughbred horses and elite human athletes but bas not yet been reported in other cases.

A third possible advantage, neglected in many treatments of this subject, is that gas mixing from locomotor-induced volume changes may reduce effective dead space, thus increasing the effective alveolar ventilation for a given total ventilation. Smail, high-frequency volume displacements can reduce effective airway dead space to zero. Such mixing will be most important at low tidal volumes where the ratio of dead space ventilation to alveolar ventilation is high. High-frequency mixing, in contrast to tidal volume assistance, would not depend on coordination of breath-stride phase relationships.

A fourth possible advantage is that synchrony may feel more comfortable, a phenomenon reported by many human runners.

If the mechanical link is strong, a possible disadvantage is reduced flexibility in changing ventilation to meet metabolic and thermal demands. If stride were to produce all or most of tidal volume, the animal would be obligated to breathe at stride frequency to take advantage of constructive interference and to avoid destructive interference. The metabolic and thermal demands on ventilation are not tightly linked to stride frequency or length, and, thus, an inability to uncouple breathing from locomotion could result in ventilation that is inappropriate for the control of gas or heat exchange. For example, during climbing (terrestrial or aerial), energetic costs increase, whereas the frequency and amplitude of the locornotor cycle may stay constant or even decrease. Similarly, the ventilatory requirement for heat loss is a function both of exercise heat production and of ambient temperature and humidity and is thus not simply related to stride frequency and amplitude.

Another possible disadvantage of strong mechanical linkage is that animals may be forced to breathe at energetically unfavorable frequency tidal volume combinations. The frequency of the locomotor cycle is high compared 
with the frequency of breathing at rest or during hypercapnia. A given alveolar ventilation requires more work at high frequency and low tidal volume combinations because dead space contribution is proportionately greater and because a significant amount of energy may be necessary to accelerate gas in the airways. Thus far, no published models have incorporated the more recently developed concept of high-frequency mixing that would change the determination of optimal frequencies nor have these models been applied to the problem of breathing at stride frequency.

\section{Proposed rnechanical linkage mechanisms}

Locomotor muscles can cause airflow by acting directly on the chest wall (diaphragm, rib cage) or by generating inertial forces that accelerate tissue masses. Several mechanical linkage mechanisms have been proposed.

lnertial displacement of the diaphragm or visceral piston mechanism. As an animal accelerates and decelerates with each stride, the abdominal viscera move relative to the spine (and thoracic cage) because they are not a rigidly attached mass. When the viscera underlying the diaphragm move along the cranial-caudal axis, they change the volume of the thoracic cavity and can cause airflow: inspiration when the diaphragm is displaced caudally by cranial acceleration of the trunk and expiration when the diaphragm is displaced cranially by trunk deceleration. In this way, the viscera may act like a piston and drive changes in lung volume (8).

Inertial displacement of the rib cage. When the spine accelerates in the cranial direction, ribs can move caudally about their articulation with the spine to decrease thoracic dorsoventral diameter ("pump handle" motion, which is limportant during resting breathing in humans) or they can move both caudally and medially about their articulations with the spine and sternum to decrease the lateral diameter of the thorax ("bucket handle" motion, which is important in quadrupeds). Both of these motions produce expiratory flows. Conversely, when the spine decelerates, ribs move cranially or laterally to increase thoracic volume and produce inspiratory flows. For a given direction of acceleration, therefore, resultant rib cage displacements generate flows in the opposite direction relative to abdominal visceral mass displacements $(5,13)$.

Spinal flexion. Flexion of the lumbar spine tends to shorten the trunk, displacing the abdominal viscera and diaphragm cranially, and causes expiratory flows. Extension tends to displace abdominal contents caudally and causes inspiratory flows (8). This mechanism assumes that the trunk crosssectional area remains relatively constant during spinal flexion and extension, which may not be true.

Rib cage loading by foretimb. Onimpactwith the ground, the forelimb of quadrupeds transmits compressive forces to the rib cage (8). The direction of respiratory flow depends on the angle of forces relative to hinge axes of the ribs, but it seems likely that limb impact forces cause expiralory flow.

Common muscle link. Chest wall muscles that function in locomotion may link breathing to locomotor cycles. These include pectoral, intercostal, and abdominal muscles. The pectoral muscles are especially 
powerful in birds, and their contraction might be expected to compress the thorax and lead to expiratory flows during wing downstroke. Activation of intercostal and abdominal muscles in quadrupeds during locomotion may not only stabilize the trunk or flex the lumbosacral joint but may also cause respiratory flows. Whether flow actually occurs, however, will depend on what other muscles are simultaneously activated.

\section{Phrasing the question}

Although it is evident even to the casual observer that some respiratory flow occurs with stride, we want to know whether the volume displaced is large enough to be important for breathing. We consider here quantifiable effects that relate directly to gas-exchange parameters (e.g., alveolar ventilation, ticial volume) and ask, How much air does a stride (or win-beat) move?

What volume of air is significant? Whether a stride-induced volume change significantly assists the respiratory muscles is most easily assessed by comparing it with tidal volume. Much smaller volumes at high frequencies might significantly improve gas exchange by mixing; the effectiveness of this action depends on the product of volume and frequency.

\section{Studies}

Investigators disagree on how much air a stride or wingbeat moves. Several investigators have proposed that locornotor activity could drive all or a substantial part of ventilation in exercising animals (e.g., Ref. 8). Mathematical models suggest that this is possible in hopping or galloping animals (4). However, actual measurements of volume change provided by stride in trotting or galloping animals range from 1 to $20 \%$, of tidal volume. Most of the measured variation is likely due to species differences. We discuss the approaches, techniques, and findings of various studies.

Mathematical models. Alexander (4) examined the plausibility of three mechanical mechanisms (visceral piston, spinal flexion, and forelimb loading) by generatino "best case scenario" models for a horse and wallaby. Using these mathernatical models to calculate the maximum ventilation that could result from various mechanisnis, he concluded that if the natural frequency of a visceral piston Ivere tuned to stride frequency, this mechanism could provide the entire tidal volume in a hopping wallaby and that spinal flexion could provide the entire tidal volume in a galloping horse. These models did not account for 1) acceleration of the rib cage that would produce volume changes that tend to negate those produced by accelerating abdominal viscera (13) and 2) trunk cross-sectional expansion that can accompany spinal flexion, which would tend to negate shortening of the trunk caused by flexion. This latter occurs in humans (14) but has not been examined in other animals.

Experimental techniques. To determine the volume of air moved by stride, we desire a measure of respiratory flow during exercise, as well as the means to determine what part of the respiratory signal is stride related. Although temperature and sound variation at the nostril or mouth can be used to infer the timing of flow, they cannot be used to quantify volume changes 
because their amplitude is not uniquely related to flow.

Respiratory flow can be measured with a pneumotachometer (e.g., in which flow through a fixed-diameter tube is inferred from the pressure drop across a known resistance or from the cooling of a heated element). One can then integrate the flow signal over time to determine volume. The timing of locomotor events is typically inferred from an accelerometer, a foot switch, or a phototransistor that alternates between light and dark over wingbeat or stride. Animals have generally been studied while running on a treadmill or flying in a wind tunnel, but there have also been a few studies of breathing in free running and flying animals.

It is not always easy to entice animals to run on treadmills or fly in wind tunnels with natural gaits or to breathe normally through respiratory equipment. Measuring respiration in exercising animals also presents several problems: for example, it is difficult to connect measuring devices (e.g., a mask with a pneumotachometer) to an animal; motion artifacts may be misinterpreted as a locomotor contribution to breathing (e.g., motion of a mask on the face may displace air through the pneumotachometer; many pressure transducers used with pneumotachometers respond to acceleration, etc.). To measure flow accurately, the mask holding the pneumotachometer must form a good seal. A tight-fitting mask, however, may interfere with breathing or locomotion by increasing the dead space and resistanceor by impeding head motion orvision. Masks may cause animals to breathe at slower frequencies (3) or higher tidal volumes, Chronic tracheostomies can provide an excellent seal without disturbing the face and head but may also interfere with normal breathing. Thus techniques that have been used to measure flow may themselves affect flow. Skill and patience are needed to find acceptable techniques for each species.

One can infer respiratory flow and volume with less encumbrance to the animal, but these measures are less direct and are subject to other artifacts. Changes in lung volume in stationary subjects have been inferred from changes in chest wall dimensions (via magnetorneters, respitrace, and radiographic reconstruction) or from changes in radiographic densities and transmission impedance.

Stride-related artifacts, however, are likely with these techniques, confounding any estimate of stride-related volume change. Magnetometers detect changes in length via the strength in magnetic field between sender and receiver coils on opposite sides of the trunk. Respitrace measures changes in cross-sectional area via changes in inductance in wire loops surrounding the trunk. Impedance pneumography measures changes in electrical characteristics of lung tissue by means of external electrodes. Because all these devices are fastened to the skin, skin motion artifacts are problematic during locomotion. In radiographic density determinations of volume change, artifacts may arise from soft tissue moving in and out of the field of measurement. Radiographic reconstruction of lung volume entails sometimes difficult judgment of lung boundaries. Estimates based on single-plane images do not account for possible opposing changes of the third dimension; simultaneous two- or three-plane images have not been done in running animals, presumably because of the difficulty and expense. 
Pleural pressure records provide information on breathing frequency but require significant analytic effort to extract flow or volume estimates. Pleural pressure changes are due to elastic, inertial, and resistive impedances; an appropriate model incorporating $\mathrm{A}$ of these impedances is required to inter volume changes from pleural pressure.

After flow or volume records have been acquired, how does one distinguish locomotorrelated signals from those that are driven by breathing muscles? When step and breathing are not synchronous, one can estimate the locomotor contribution from fluctuations in the flow signal that correspond in time to step. In some cases, these fluctuations are visible by eye. For example, Fig. 1 shows a record of airflow in an exercising dog; the flow fluctuation associated with one step of the trot is easily distinguished.

Step-related flow can be quantified more rigorously by ensemble averaging the flow signals recorded during many steps. A trigger point is selected from each cycle of the locomotor signal. The trigger points of many step cycles are then aligned on the same time axis, superimposing their corresponding flow records. When the flow records are averaged, they produce a trace that represents flow during an average step cycle. if steps are uniformly distributed throughout breathing cycles, then flows due to the act of breathing will average zero. If we assume that the mechanical effects of stride events add linearly to the effects of respiratory muscles, we may regard the residual ensemble-averaged flow as an estimate of step-related flow. if steps are not uniformly distributed, the resulting average flow record is biased and may overestimate stride contribution. If the effects do not add linearly (e.g., if stride systematically changes respiratory muscle activation), the resulting average flow record may either overestimate or underestimate stride contribution.

Experimental results. Locomotor contribution to tidal volume. Several studies have reported flow measured at the airway opening during locomotion. Ensemble averaging shows that the locomotor-related contribution is $<20 \%$ of the tidal volume. In starlings, although strong coupling might be expected because the pectoralis muscles insert on the sternum and exert force during wing downstrokes, wingbeat accounted for 3-11\% of the tidal volume (6). Similar results were also found in quadrupeds. In galloping horses, the steprelated volume change Ivas $<20 \%$ of tidal Volume (12). In trotting dogs, it was 3-16\% (7), consistent with data of Ainsworth and co-workers shown in Fig. 1. In hunians, whose bipedal posture and gait are expected to be associated with a greater mechanical independence (8), the mechanical contribution of stride to tidal volume during walking and running was $1-2 \%$ (5). In addition to these estimates of the total locomotor contribution to tidal volume, the contributions of various proposed mechanisms have been evaluated qualitatively in several studies and are summarized below.

Viscfral piston. Bramble and Jenkins (9) compared flows measured with a pneumotachometer in an intentionally loosely fitted mask with a twodimensional cineradiographic analysis of diaphragm volume displacement and concluded that inertial displacements of the visceral piston appeared to be the primary driver of diaphragm displacement in trotting dogs. Young et al. (15) measured tidal volume with a pneumotachometer-mask combination and trunk 
accelerations from high-speed films and concluded that the inertially driven visceral piston could not be driving breathing because the phase relationship between volume and acceleration was not appropriate. Ainsworth and coworkers found that during every breath cycle, inspiratory swings in transdiaphragmatic pressure corresponded with phasic diaphragmatic electromyogram activity in trotting dogs (3), as well as trotting and galloping horses (2), and thus concluded that inspiratory motions of the diaphragm resulted from muscle contraction rather than from inertial forces.

Spinal flexion. Young and co-workers (15) used a model that incorporated trunk shortening (but not cross-sectional changes) to compare lumbosacral flexion mesured from cinefilms with tidal volume. They concluded that, at speeds of $9 \mathrm{~m} / \mathrm{s}$, spinal flexion in galloping horses was of the proper magnitude and phase to drive i substantiai part of tidal volume.

Forelimb Bramble and Jenkins (9) observed, via two-dimensional cineradiography, that loading by forelimbs produced prominent unilateral inward deformation of the rib cage in the region of limb articulation in trotting dogs. Although they did not assess the effect of this compression on lung volume, the deformation appears large enough to cause an appreciable expiratory effect.

Common muscle links. Several respiratory muscles (e.g., interosseous intercostal and abdominal muscles) also have locomotor functions, inferred from correlation of their phasic electromyogram activity with the locomotor cycle when breathing and locomotion were uncoupied in trotting dogs $(3,11)$. One cannot deduce the mechanical effect of a muscle on the respiratory system from measures of electrical activity; therefore, the effectiveness of this link has yet to be assessed.

\section{Perspectives}

Because structures of the chest wall serve both respiratory and locomotor functions, opportunities exist for both conflict and cooperation. These opportunities may constrain both processes. Such functional constraints are especially apparent in the lizardIguana iguana, which actually reduces lung ventilation at high locomotor speeds (10). The evolution of proper neurological coordination was apparently necessary to enable animals to breathe effectively while running or flying. In addition, mechanical linkages may have also facilitated a recluced cost or a higher level of performance of one or both of these functions.

How much energy could be saved by mechanical links between locomotion and breathing? During sustainable levels of aerobic exercise (e.g., trot or slow gallop), ventilation consumes only $5 \%$ of the total energy budget, and the required ventilation is well within the capacity of the respiratory muscles. Measurements of respiratory volume associated with stride suggest that less than one-fifth of the work of breathing can be provided by locomotor muscles, yielding $<1 \%$ net energy savings. On the other hand, a few animals encounter limitation of ventilatory capacity during heavy exercise, resulting in arterial oxygen desaturation; in these animals, a $20 \%$ increase in ventilatory capacity could be quite important in increasing performance, even if it bas little effect on overall efficiency. 


\section{References}

1. Aaron, F. A., K. C. Seow, B. D. Johnson, and J. A. Dempsey. Oxygen cost of exercise hyperpnea: implications for performance. J. Appl. Physiol. 72: 1818-1825, 1992.

2. Ainsworth, D. M., S. W. Eicker, M. E. Nalevanko, N. G Ducharme, R. P. Hackett, and K. Snecclen. The effect of exercise on diaphragmatic activation in horses. Respir. Physiol. 106: 35-46, 1996.

3. Ainsworth, D. M., C. A. Smith, K. S. Henderson, and J. A. Dempsey. Breathing during exercise in dogs-passive or active? J. Appl. Physiol. 81: 586-595, 1996.

4. Alexander, R. McN. On the synchronization of breathing with running in wallabies and horses (Equus caballus). 1. Zool. (Lond.) 218: 69-85, 1989.

5. Banzett, R. B., J. Mead, M. B. Reid, and G. P. Topulos. Locomotion in men has no appreciable mechanical effect on breathing. J. Appl. Phlysiol. 72: 19212-1926, 1992.

6 Banzett, R. B., C. S. Nations, N. Wang, J. P. Butler, and L. Lehr. Mechanical independence of wing beat and breathing in starlings. Respir. Physiol. 89: 27-36, 1992.

7. Birlenbach, U. S., R. B. Banzett, S. H. Loring, and D. R. Carrier. A modest fraction of tidal volume is step-related in trotting dogs (Abstract). Physiologist 37: A-51, 1994.

8. Bramble, D. M., and D. R. Carrier. Running and breath ing in mammais. Science 2 19: 251 -2 $56,1983$.

9. Bramble, D. M., and F. A. Jenkins, Jr. Maminalian locomotor-respiratory integration: implications for diaphragmatic and pulmonary design. Science 262: 235) 40 1991.

10. Carrier, D. R. Lung ventilation during walking and running in tour species of hzards. Exp. Biol. 47: 33-42, 1987.

11. Carrier, D. R. Function of the intercostal muscles in trotting dogs: ventilation or locomotion? J. Exp. Biol. 199:1455-1465,1996.

12. Frevert, C. W., C. S. Nations, H. J. Seeherman, S. H. Loring, and R. B. Banzett. Airflow associated with stride in the horse (Abstract). Physiologist 33: A-83, 1990.

13. Liu, S., T. A. Wilson, and K. Schreiner. Gravitational torces on the chest wall. J. Appl. Physiol. 70: 15061510,1991.

14. Mead, J., 1. C. Smith, and S. H. Loring, Volume displacements of the chest wall and their mechanical significance. In: The Thorax, edited by C. Roussos and P. T. Macklem. New York: Dekker, 1985, p. 369-392.

15. Young, 1. S., R. McN. Alexander, A. J. Woakes, P. J. Butler, and L. Anderson. The synchronization of ventilation and locomotion in horses (Equus caballtis). J. Exp. Biol. 166: 19-31, 1992. 